\title{
Interdisciplinary Education - A Predator-Prey Model for Developing a Skill Set in Mathematics, Biology and Technology
}

\author{
Quay van der Hoff \\ Department of Mathematics and Applied Mathematics, University of Pretoria, South Africa \\ Private Bag Pretoria 0002 \\ quay.vanderhoff@up.ac.za
}

\begin{abstract}
The science of biology has been transforming dramatically and so the need for a stronger mathematical background for biology students has increased. Biological students reaching the senior or post-graduate level often come to realize that their mathematical background is insufficient. Similarly students in a mathematics programme, interested in biological phenomena find it difficult to master the complex systems encountered in biology. In short, the biologists do not have enough mathematics and the mathematicians are not being taught enough biology.
\end{abstract}

The need for interdisciplinary curricula that includes disciplines such as biology, physical science, information technology, and mathematics is widely recognized, but has not been widely implemented. In this paper it is suggested that mathematical biology students develop a skill set of biology (ecology), mathematics, modeling and technology to encourage working across disciplinary boundaries. To illustrate such a skill set a predator-prey model that contains self-limiting factors for both predator and prey, is suggested. The general idea of dynamics, as described by differential equations is introduced and students are encouraged to discover the applicability of this approach to the dynamics of more complex biological systems. 
The level of mathematics and technology required is not advanced; therefore it is ideal for inclusion in a senior-level or introductory graduatelevel course for students interested in mathematical biology in which three important disciplines - biology, mathematics and technology - come together to develop a skill set for prospective researchers.

Keywords: Mathematical biology, predator-prey, interdisciplinary, mathematical modeling, ordinary differential equations, computer algebra system, technology.

\section{Introduction}

The science of biology has been transforming dramatically with advances in biological understanding, experimental techniques and computational analysis and recent reports have called for change in how undergraduate mathematics is approached for students in biology $[1,2]$. It has become apparent that no single discipline can fully address the challenges of new frontiers in biology [3], and as such the average life science student is not exposed to enough areas in mathematics [4]. The use of mathematics in biological research is taking on many forms: statistics in experimental design; pattern seeking in bioinformatics; models in evolution, ecology and epidemiology and many more [5]. The need for knowledge of quantitative biology is even more evident in systems biology, where at molecular level this involves building mathematical models and understanding complex processes such as gene regulation, protein-protein interactions and metabolism $[6,7]$.

Biological sciences have been revolutionized, not only in the way research is conducted but also in how research findings are communicated among professionals and to the public. Yet, the undergraduate programs that train biology researchers remain much the same as they were before these fundamental changes came about [1, 8]. In many universities, majors in mathematics, physics, chemistry and engineering are taught separately from those hoping to be biologists or physicians [1]. These students traditionally do only one or two semesters of mathematics, often not with much depth, since mathematics is not their primary interest. It is only once they get to senior-level or 
post-graduate level that they realize that their mathematical background is insufficient. Similarly, students in a mathematics programme who become interested in biological phenomena find it difficult to master the complex systems encountered in biology. In short, the biologists do not have enough mathematics and the mathematicians are not being taught enough biology. These barriers to communicate between disciplines have become more and more evident.

Calls for improvement in interdisciplinary education date from as far back as 1976, with Robert May's [8] plea for the introduction of difference equations, used in, amongst other, ecological modeling, into elementary mathematics courses. Bialek and Botstein [1] advocate an integrated introductory curriculum in which mathematics, physical sciences and biology is introduced simultaneously. They suggest that disciplines should be introduced at a high level of sophistication, because a large part of the goal is to show students how each discipline contributes to understanding the phenomena of life. Hastings and Palmer [9] advocate that the training of scientists with expertise in both biology and mathematics must be promoted.

Although the need for a strong interdisciplinary curriculum that includes disciplines such as biology, physical science, information technology, and mathematics is widely recognized, it has not been widely implemented. These changes will only become a reality once the different faculties decide to collaborate and a serious paradigm shift takes place on how such a curriculum is structured.

How then can this gap between mathematics, biology and other related disciplines be bridged? Eager, Pierce and Barlow [4] suggest first developing a skill set of mathematics, mathematical modeling and technology to encourage working outside disciplinary boundaries. Practically this would mean identifying a biological (or other) problem that could benefit from mathematical or theoretical treatment. The next step would be to create a model to make predictions about the biological system and to understand the uncertainties associated with these predictions and finally develop the skills and ability to analyze the results obtained from the mathematical model. 
An excellent vehicle for developing such a skill set is a system of differential equations, such as the Lotka-Volterra predator-prey model. The model arises in many settings in biology, ecology, economics and social sciences and although these models are simple they exhibit a surprising array of dynamical behavior.

In this paper, students are guided through the modeling process, classification of equilibrium points and finally using technology to visualize the possible outcomes. In this way three important disciplines - biology, mathematics and technology - come together to develop the skill set of prospective researchers. The general idea of dynamics, as described by differential equations, is conveyed and students are encouraged to discover the applicability of this approach to the dynamics of more complex biological systems.

The model, in essence, a classical Rosenzweig-MacArthur type, although simple, displays rich dynamical behavior. Students must apply a numerical investigation to determine the conditions under which the model possesses either a stable equilibrium or limit cycles, or under which conditions the uniqueness of the equilibrium cannot be guaranteed. In the latter case a Computer Algebra System (CAS) is used to investigate the long term behaviour of trajectories in order to predict the nature of the unique or multiple equilibrium points. The investigation includes defining and plotting the separatrices and trajectories to gain insight into the dynamics of the system.

In most cases there is but a single critical value, an attracting spiral point suggesting a stable population pair or an unstable node, resulting in a unique limit cycle. The critical values of the system are related to the roots of a cubic polynomial and it is only when the cubic has multiple positive roots that the system becomes unstable. Thus the analysis of equilibria entails investigating a well-known cubic polynomial. The level of mathematics and technology required is not advanced; therefore it is ideal for inclusion in a seniorlevel or introductory graduate-level course for students interested in mathematical biology. In addition, by introducing any available CAS, such as Mathematica [10], interesting phenomena of the dynamics of such a modeled system can be observed. 


\section{The proposed mathematical model}

The point of departure is that the student has some basic knowledge of predator-prey models and expanding the model contributes to understanding the reasoning behind and ecological basis for the inclusion and form of each of the terms in the suggested model.

The model is, in essence, a classical Rosenzweig-MacArthur type model (Rosenzweig and MacArthur 1963), [11] but with an added function $h(y)=\left(1-\frac{y}{L}\right)$, which is called the population saturation of the predator. Refer to Van der Hoff [15] for the necessary assumptions and a more in-depth discussion on the development of the model.

The model is given by:

$$
\begin{aligned}
& \dot{x}=r x\left(1-\frac{x}{K}\right)-\alpha\left(\frac{x y}{x+H}\right) \\
& \dot{y}=-\gamma y+\varepsilon\left(\frac{x y}{x+H}\right)\left(1-\frac{y}{L}\right)
\end{aligned}
$$

where $x(t)$ denotes the number of prey and $y(t)$ denotes the number of predators at given time $t$.

System (1) contains the conventional logistic term $r x(1-x / K)$ and a Holling type II functional response $\alpha x /(x+H)$. The constant $L$ is introduced as the saturation population of the predator in the same way as the $K$ represents the carrying capacity of the prey. The intrinsic growth rate of prey is represented by $r$ while $\gamma$ is the mortality rate of predator. The attack coefficient $\alpha$, also called the capturing rate, is the rate at which a predator can search out its prey, while $\varepsilon$ is the conversion rate, that is in simple terms, the predator's efficiency in turning food into offspring. The constant $H$ contained in the Holling type II functional response is the half-capturing saturation constant. The model parameters are all assumed to be positive.

Since the proposed model contains self-limiting factors for both predator and prey and thus has bounded solutions $x(t)$ and $y(t)$ in the population quadrant. The inclusion of $h(y)$ is effectively saying that the predators are becoming less efficient at converting prey biomass to predator biomass when there are more predators around. This is validated by a 
study conducted by Schmidt and Mech [12] on the optimal wolf pack size. They asked the question: Does living in large groups facilitate the acquisition of large prey? In other words, will a pack of eight wolves be twice as successful as a pack of four? If this is so, then the food acquired per wolf should increase as the pack increases.

However, they found no evidence to indicate that an increased pack size will lead to an increased food supply. In fact, all the data they used suggested that there was less food per wolf as pack size increased. They use the term "optimal pack size" - which is equivalent to population saturation $L$ in the suggested model. The interested reader is referred to Schmidt and Mech [12] for more detail.

The relevance of having bounded solutions is reflected in the Poincaré-Bendixon Theorem [13], which predicts that if a single equilibrium point exists within this bounded region, then it is either an attracting spiral point, suggesting a stable population pair, or an unstable node, resulting in a unique limit cycle. If uniqueness cannot be guaranteed the model becomes unstable, but the analysis of the behaviour of solutions leads to some interesting phenomena.

\section{Mathematical Analysis of the Model}

\subsection{Analysis of equilibrium points}

For the following analysis the student needs to be familiar with basic concepts related to nonlinear systems of differential equations. Students will be expected to master the concepts of equilibrium point, linearization, Jacobian, stability, Poincaré-Bendixson theory, limit cycles, etc. Investigation of stability of the model reduces to eigenvalue analysis to determine the nature of the equilibrium points. The relevance of the latter should be highlighted as this indicates whether the species will coexists or if extinctions will occur. These topics are dealt with in many textbooks such as Zill and Cullen [13].

An alternative approach that could be introduced at this stage is a useful tool for the analysis of nonlinear systems: nondimensionalization, or rescaling [14]. This is a process for transforming a series of equations (usually ODEs or PDEs) to dimensionless (i.e. unitless) forms by rescaling the model variables. In the process the number of parameters is reduced but the dynamics of the system of equations remain the same. In the case of 
System (1) nondimensionalization will reduce the number of parameters from 7 to 4 , but for our example we will continue with the original number of parameters.

It is convenient to use eigenvalue analysis by determining the signs of the determinant and trace of the Jacobian matrix. For example, an equilibrium point $E^{*}\left(x^{*}, y^{*}\right)$ of System (1) is unstable if both the determinant and the trace of the Jacobian matrix are positive [13].

It is easily shown that the determinant of the Jacobian matrix is given by

$$
\Delta=\left(\frac{-\gamma x(H-K+2 x)}{K(H+x)}\right)\left(\frac{-\gamma y}{L-y}\right)+\varepsilon\left(\frac{H}{(H+x)^{2}}\right)\left(\frac{x}{(x+H)}\right)\left(1-\frac{y}{L}\right) y
$$

and the trace is given by

$$
\tau=\frac{-r x(H-K+2 x)}{K(H+x)}-\frac{\gamma y}{L-y} .
$$

To determine the nature of each equilibrium point, the sign of both the trace and determinant need be determined in each of the equilibrium points.

To determine the equilibrium points of System (1), consider

$$
r x\left(1-\frac{x}{K}\right)-\alpha\left(\frac{x y}{x+H}\right)=0 \text { and }-\gamma y+\varepsilon\left(\frac{x y}{x+H}\right)\left(1-\frac{y}{L}\right)=0 .
$$

From this follows that the system has trivial equilibrium points at $E_{0}(0,0)$ and $E_{1}(K, 0)$ neither situated in the population quadrant and indicating extinction of one or both species.

The non-trivial equilibrium points, that is those within in the population quadrant that could lead to co-existence of both species, are defined by the two simultaneous equations, the $x$-nullcline

$$
y=\frac{r}{\alpha}\left(1-\frac{x}{K}\right)(H+x)
$$

and the y-nullcline,

$$
y=-\frac{L}{\varepsilon x}(H \gamma+x \gamma-x \varepsilon)
$$


A graphical analysis of these nullclines can be a powerful tool in investigating the nonlinear dynamics of System (1). For example, if the vertex of the $x$-nullcline (4) has a negative $x$-coordinate it precludes having more than one equilibrium solution in the first quadrant. This comes down to the condition that $a=(H-K)>0$, which will be referred to in Section 3.3. Further exploration into nullclines and the interpretation thereof should be encouraged.

When equated and simplified, the nullclines deliver the $x$-values of the equilibrium points. That is, the $x$-values can be found by solving $P(x)=0$ where

$$
P(x)=x^{3}+(H-K) x^{2}+\frac{K L \alpha \varepsilon-K L \alpha \gamma-H K r \varepsilon}{r \varepsilon} x-\frac{H K L \alpha \gamma}{r \varepsilon} .
$$

In simple terms, the cubic equation $P(x)=0$ needs to be solved where

$$
P(x)=x^{3}+a x^{2}+b x-c
$$

with $a=(H-K), b=(K L \alpha \varepsilon-K L \alpha \gamma-H K r \varepsilon) / r \varepsilon$ and $c=H K L \alpha \gamma / r \varepsilon$.

A unique equilibrium point in the population quadrant will exist if $P(x)$ possesses one, and only one, intercept (root) with the positive $x$-axis. Note that $c>0$, since all the parameters of System (1) are assumed to be positive. Let $a$ and $b$ be arbitrary.

For all values of $a$ and $b$ the function $P(x)$ will entertain only one positive root, except for one case, namely if $b>0, a<0$ and $a^{2}>3 b$. In the latter case, since $b>0$, and $\sqrt{a^{2}-3 b}<|a|$, it is clear that $P(x)$ will have two critical points with positive $x$-values,

namely $x_{1}=\left(-a+\sqrt{a^{2}-3 b}\right) / 3$ and $x_{2}=\left(-a-\sqrt{a^{2}-3 b}\right) / 3$, yielding the possibility of either one or three positive roots. If the signs of $P\left(x_{1}\right)$ and $P\left(x_{2}\right)$ are the same, then $P(x)$ possesses a unique positive root. However, the more interesting scenario is when the signs of $P\left(x_{1}\right)$ and $P\left(x_{2}\right)$ differ, in which case the cubic will present with three positive roots, hence System (1) will have three distinct equilibrium points.

\subsection{Numerical Analysis}

Experience in applying a CAS system is beneficial in many research areas. The student is encouraged to compare the analytical findings to visual exploration. 
Using the CAS system Mathematica choose parameter values that satisfy all the necessary assumptions to obtain a visual illustration of these scenarios are given below.

\subsubsection{Unique equilibrium}

If System (1) has a unique equilibrium in the bounded region, then the PoincaréBendixon Theorem predicts either an attracting spiral point, suggesting a stable population pair, or an unstable node, resulting in a unique limit cycle. In both cases the species will coexist.

As an example, choose parameter values $r=12, \varepsilon=4, \gamma=1.5, H=6.2$ and $\alpha=20$. In Figure 1, the trajectory $(x(t), y(t))$ is depicted for varying values of the carrying capacity $K$ and the population saturation $L$.

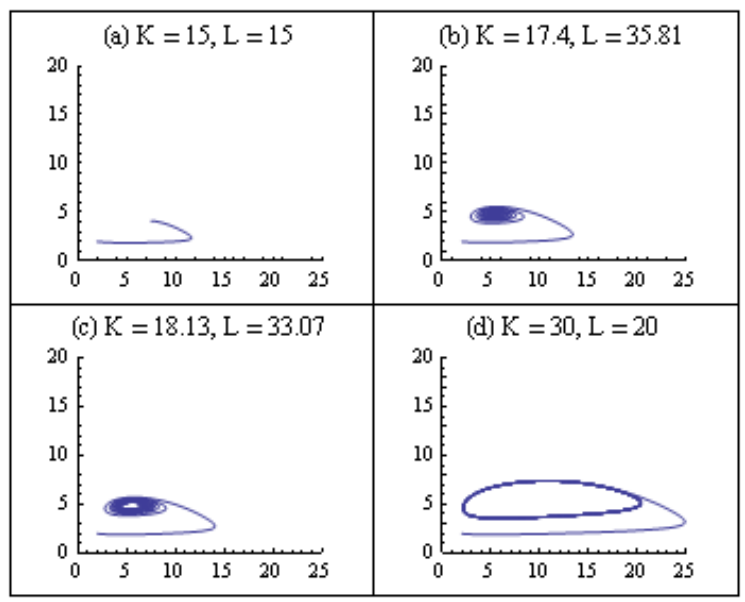

Figure 1:The trajectory $(x(t), y(t))$ for varying values of $K$ and $L$

In Figure 1(a), it can be seen that if $K=15$ and $L=15$ then the corresponding equilibrium point $(7.5773,4.0906)$ is stable. In Figure $1(\mathrm{~b})$, if $K=17.4$ and $L=35.81$ the equilibrium point is an attracting spiral. If $K=18.13$ and $L=33.07$ the beginning of a limit cycle is evident as the bifurcation occurs. This is illustrated in Figure1(c). In Figure1 (d), with $K=30$ and $L=20$, the limit cycle can clearly be seen.

\subsubsection{Multiple equilibrium points}

Choose parameter values $r=2.4, K=10, L=5, \alpha=1, H=1, \varepsilon=10$ and $\gamma=1$.

The conditions $b>0, a<0$ and $a^{2}>3 b$ are satisfied, resulting in 


$$
P(x)=x^{3}-9 x^{2}+8.75 x-2.0833 \text {. }
$$

The cubic $P(x)$ has three positive roots at $x_{1}=0.3813, x_{2}=0.6890$ and $x_{3}=7.9297$, hence the system has three non-trivial equilibrium points in the population quadrant. System (1) has trivial equilibrium points $E_{0}$ and $E_{1}$ and therefore the system has five equilibrium points in total. The trace and determinant of each equilibrium point can be determined by using Equations (2) and (3). Table I contains a summary of the detail needed to discuss the nature of these equilibrium points.

\begin{tabular}{|c|c|c|c|c|}
\hline Equilibrium & $x$ & $y$ & Trace $(\tau)$ & Determinant $(\Delta)$ \\
\hline$E_{0}$ & 0 & 0 & 1.4 & -2.4 \\
\hline$E_{1}$ & 10 & 0 & 5.69091 & -19.4182 \\
\hline$E_{2}$ & 0.381312 & 3.18874 & -1.21476 & 0.71044 \\
\hline$E_{3}$ & 0.689005 & 3.77432 & -2.33313 & -0.974846 \\
\hline$E_{4}$ & 7.92968 & 4.43695 & -9.34203 & 11.5755 \\
\hline
\end{tabular}

Table I: Trace and determinant of each equilibrium point

The trivial equilibrium points $E_{0}$ and $E_{1}$, both situated on the population axes, are saddles since they have negative determinant values. Equilibrium points $E_{2}$ and $E_{4}$ have positive determinants and negative traces and therefore are classified as stable. The equilibrium point $E_{3}$ is classified as a saddle, since both the determinant and the trace are negative. Using Mathematica, these saddles and the stable equilibrium points are shown in the vector field of the System (1) as depicted in Figure (2). 


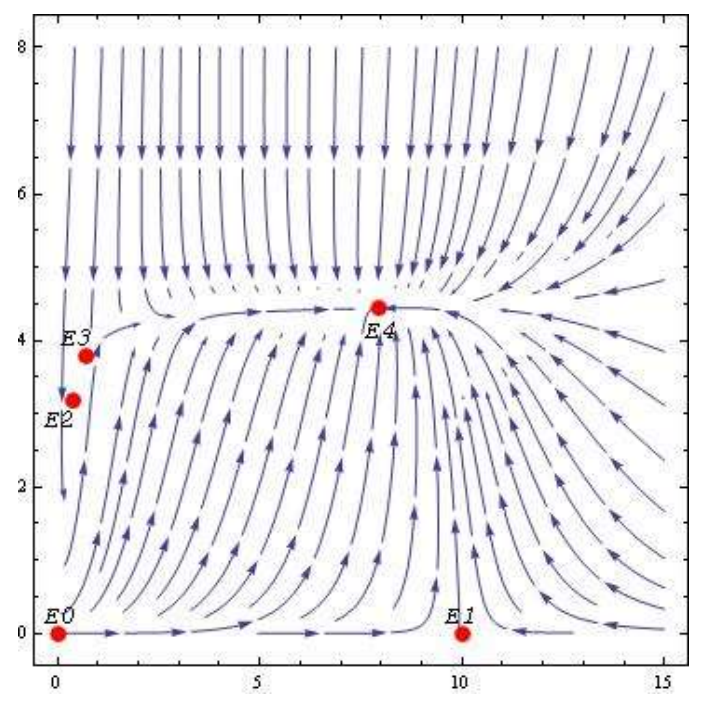

Figure 2: Vector field of System (2)

To understand the behaviour of the trajectories in the vicinity of $E_{2}$ and $E_{3}$ it is helpful to consider the following: Since $E_{3}=\left(x_{3}, y_{3}\right)$ is a saddle point, it should be possible to find the separatrices. We refer to a method described by Fay and Joubert [16] on how to use numerical investigations to plot the separatrices, even though it is not possible to determine the equations of the separatrices.

Solve the System (1) using initial conditions

$$
\begin{aligned}
& x(0)=x_{3} \pm 5 \times 10^{-6}, y(0)=y_{3} \text { and } \\
& x(0)=x_{3}, y(0)=y_{3} \pm 5 \times 10^{-6} .
\end{aligned}
$$

This results in four solutions of which the trajectories, represented in both forward and backward time, are depicted in Figure 3, showing the separatrices and all five equilibrium points. 


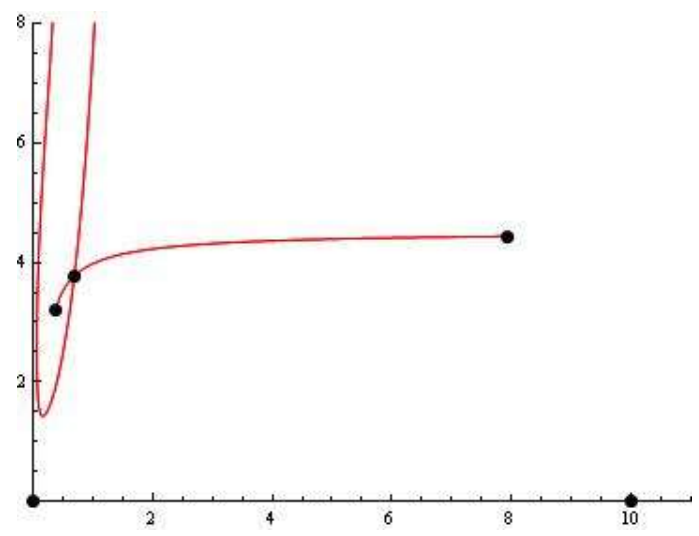

Figure 3: The separatrices of the saddle E3

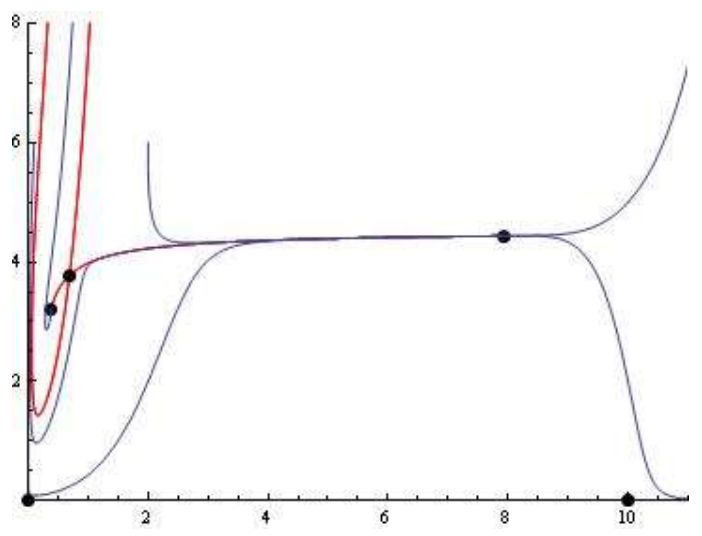

Figure 4: Phase portrait of System (2)

By judicious choice of initial conditions and solving in both forward and backward time, a phase portrait can be generated showing representative behaviour of the model. This is shown in Figure 4.

The separatrices divide the plane into two regions; one is inside the parabolic- appearing curve and the other is the region outside. If an initial condition is chosen on the outside of the parabola, the trajectory is attracted to the node $E_{4}$, while if the initial condition is on the inside, the trajectory is attracted to the spiral point $E_{2}$. The two trajectories connecting $E_{2}$ to $E_{3}$ and $E_{3}$ to $E_{4}$ together form a heteroclinic path.

Similar numerical analysis can be carried out to classify the equilibrium points and produce a phase portrait by varying the parameter values such as $r=12, K=80, L=20, \alpha=20, H=4, \varepsilon=4$ and $\gamma=1.6$. Also worth exploring is the case where the cubic $P(x)$ has three roots but one root is repeated for example use parameter values $r=2.4, K=19, L=5.0268, \alpha=25, H=10, \varepsilon=10$ and $\gamma=0.011350$.

\subsection{Summary of findings}

The model (1) supports the feature that the solutions are bounded from the onset by the carrying capacity of the prey and the population saturation of the predator. This makes applying Poincaré-Bendixson theory possible as long as the equilibrium point in the population quadrant is unique. 
System (1) possesses a unique equilibrium point in the population quadrant at $0<x^{*}<K$ and $y^{*}=r / \alpha\left(1-x^{*} / K\right)\left(H+x^{*}\right)$ if

(i) $b \leq 0$, or (ii) $a^{2}-3 b \leq 0$, or (iii) $b>0$ and $a>0$

where $a=(H-K), b=(K L \alpha \varepsilon-K L \alpha \gamma-H K r \varepsilon) / r \varepsilon$ and $c=H K L \alpha \gamma / r \varepsilon$.

In this case, System (1) is robust and results in system stability, yielding equilibrium values which lead to either limit cycles or attracting spiral points. However, no general conclusion may be drawn regarding the number or nature of the equilibrium points in the case where $b>0, a<0$ and $a^{2}>3 b$ and further investigation is necessitated for each individual case.

Lastly, as the dynamics of the model are influenced by the parameters $a, b$ and $c$, we need to answer questions such as: what are the physical interpretations of these parameters and is it reasonable that the dynamics should be influenced by them. Further investigations of the model should help in the understanding of what is driving the dynamics from a biological point of view.

\section{Conclusion}

The aim of this paper is to introduce an example which can be used to develop a skill set for prospective mathematical biology students. Students are guided through the modeling process by means of a system of nonlinear differential equations that describe an ecological situation. The terminology and reasoning behind and ecological basis for the inclusion and form of each of the terms in the model form an important component in mathematical modeling.

The methods of solution, applications and theory of ordinary differential equations are introduced. The classification of equilibrium points and relevancy of having a unique equilibrium point is interpreted in the ecological context.

Finally, using technology the numerical calculations are simplified and using a CAS the possible long term behavior of the system can be visualized.

This skill set acquired should be of value to students with either a mathematical or biological background entering a research career. The gap that exists in these important disciplines can be transcended by analyzing similar examples and students should be 
encouraged to discover the applicability of this approach to the dynamics of more complex biological systems.

\section{References}

[1] Bialek W, Botstein D. Introductory Science and Mathematics Education for $21^{\text {st }}$ Century Biologists. Science. 2004;303:788-790.

[2] BIO 2010: Transforming Undergraduate Biology Education to Prepare Research Scientists for the $21^{\text {st }}$ Century. The National Academies, National Academies Press, Washington, D.C. 2010.

[3] Feser J, Vasaly H, Herrera J. On the edge of Mathematics and Biology Integration: Improving Quantitative Skills in Undergraduate Biology Education. CBE-Life Sciences Education. 2013;12:124-128.

[4] Eager EA, Pierce J, Barlow P. Math Bio or Biomath? Flipping the mathematical biology classroom, Letters in Biomathematics. 2014;2:139-155.

[5] May RM. Uses and Abuses of Mathematics in Biology. Science. 2004;303:790-792.

[6] Robeva R, Laubenbacher R. Mathematical Biology Education: Beyond Calculus. Science. 2009;325:542-543.

[7] Kirk PDW, Babtie AC, Stumpf MPH. Systems biology (un)certainties. Science. 2015; 350(6259):386-388.

[8] May RM. Simple mathematical models with very complicated dynamics. Nature. 1976; 261:86-93.

[9] Hastings A, Palmer MA. A bright future for Biologists and Mathematicians? Science. 2003;299:2003-2004. 
[10] Wolfram S. The Mathematica Book, 3rd ed. Wolfram Media /Cambridge University Press, New York. 1999.

[11] Smith H. L. The Rosenzweig-MacArthur predator-prey model [Internet]. Lecture Notes, School of Mathematical and Statistical Sciences, Arizona State University [cited 7 December 2016]. Available from: http://math.la.asu.edu/ halsmith/Rosenzweig.pdf

[12] Schmidt PA. Mech, LD. Wolf Pack Size and Food Acquisition. Am. Nat. 1997; 150(4):513-517.

[13] Zill DG, Cullen MR. Advanced Engineering Mathematics. Second ed. Boston, Massachusetts: Jones and Bartlett Publishers. 2000.

[14] Hall R. Nondimensionalization for dummies [Internet]. 2012 [cited 7 December 2016]. Available from: https://daphnia.ecology.uga.edu/ceesg/wpontent/uploads/2014/01/nondim_notes.pdf

[15] Van der Hoff Q. Stability of a predator-prey model with predator population saturation. PhD Thesis. Thswane University of Technology, Pretoria. 2013.

[16] Fay TH, Joubert SV. Separatrices. International Journal of Mathematical Education in Science and Technology. 2010;41(3):412-418. 